\title{
COMPARATIVE EVALUATION OF THE PUSH-OUT BOND STRENGTH OF NANO-FORMULATIONS OF MTA AND PORTLAND CEMENT
}

\author{
Yousra Mohamed Nashaat* and Nehal Nabil Roshdy**
}

\begin{abstract}
Purpose: To investigate and compare the push-out bond strength of nano-formulation of ProRoot MTA (NPMTA) and Portland cement (NPC) with commercially available ProRootMTA (PMTA) and Portland cement (PC) aiming to develop a new root-end filling/ perforation repair .material

Methods: Forty root-dentin slices prepared from freshly extracted single-rooted teeth were according to the tested cements: PMTA, PC, NPMTA ( 10 = randomly allocated into four groups (n and NPC. The root canal lumens were instrumented and filled with the test materials. The Universal testing machine was used for push-out bond strength evaluation. The maximum force employed at the time of dislodgment was recorded and the specimens were inspected under scanning electron magnification to detect the nature of the bond failure. Kruskal-Wallis test was microscope at $\mathrm{x} 25$ applied for the comparison between the groups. Mann-Whitney $\mathrm{U}$ test was used for pairwise .comparison
\end{abstract}

Results: The mean push-out bond strength \pm standard deviation values of PMTA, PC, NPMTA respectively. There was no $0.43 \pm 2.22$ and $0.39 \pm 1.1,0.34 \pm 0.91,0.4 \pm 1.33$ and NPC were but the $(0.529=$ statistically significant difference between the means of PMTA, PC and NPMTA ( $p$ Under stereomicroscope.$(0.012=$ mean of NPC was significantly higher than the three groups $(\mathrm{p}$ PMTA, PC and NPMTA groups showed a majority of adhesive failure, while NPC showed a .majority of mixed type failure

Conclusions: The force needed for NPMTA displacement is similar to PMTA and PC and significantly lower than the force required for NPC displacement

.KEYWORDS: Adaptation, Dislodgement, Leakage, Nano Materials, Root End Sealing

\footnotetext{
* Associate Professor of Endodontics, Endodontic Department, Faculty of Dentistry, October 6 University, Cairo, Egypt. ** Associate Professor of Endodontics, Endodontic Department, Faculty of Dentistry, Cairo University, Cairo, Egypt.
} 


\section{INTRODUCTION}

Mineral Trioxide aggregate (MTA) has been introduced by Torabinejad et al. (1) in 1990s, it showed great success when used as perforation repair and root-end filling material ${ }^{(2)}$. The major components of MTA matched the primary components of Portland cement (PC). MTA is composed of a mixture of Portland cement (75\%), bismuth oxide (20\%), gypsum $(5 \%)$ and small amount of $\mathrm{SiO} 2, \mathrm{CaO}, \mathrm{MgO}, \mathrm{K} 2 \mathrm{SO} 4$ and $\mathrm{Na} 2 \mathrm{SO} 4$. The four main compounds in $\mathrm{PC}$ are: dicalcium silicate, tricalcium silicate, tricalcium aluminate, and tetracalcium aluminoferrite. The strength of the hydrated cement is attributed to the silicates. Many researchers have focused on the similarities between MTA and Portland cement (PC) to present a cheaper substitute for the MTA. ${ }^{(3)}$

Meanwhile, nanotechnology has revolutionized all aspects of healthcare including the dentistry. It is the science of developing functional materials with new properties by using the nanometer scale of the materials. ${ }^{(4)}$ According to the European Commission, nano-form material is a term given to any natural or manufactured material containing particles, in an unbound or an aggregate state where, $50 \%$ or more of the particles has an external dimension of size range $1-100 \mathrm{~nm}$ " (5) . Studies have verified that the improvement of the physical and chemical properties of calcium-silicate based cements is directly related to the minute particle size and the high surface area which causes faster hydration and less porosity ${ }^{(6,7)}$. Hence, it was a logic step to produce functional materials in range of 0.1$100 \mathrm{~nm}$ through utilizing the nanotechnology as nanoparticles with their exceptional small particle size and uniform distribution of constituents can enhance the physical properties of the materials. ${ }^{(8,9)}$.

The marginal adaptation and bond strength of Calcium-silicate based cements to dentine is of extreme importance in clinical practice. The bond strength of the restorative material to dentine is assessed by the push-out strength test. Thus, the aim of this invitro study was to assess the push-out bond strength of Nano-formulated MTA and Nanoformulated PC in comparison with the ProRoot MTA and PC aiming to develop a novel root-end filling/perforation repair material. The tested null hypothesis was that there is no significant difference in the push-out bond strength of the four tested materials.

\section{MATERIALS AND METHODS}

\section{Sample size:}

Based on Saghiri et al 2013 (8) and using oneway ANOVA test, assuming an effect size of 2.45, an estimated type I error of 0.05 and power of 0.8 , a minimum total sample of 8 teeth was required to detect a statistically significant difference between the four tested groups. The sample size was calculated using G Power software version 3.1.9.2.

\section{Manufacturing of the cements}

The Research Ethics Committee of Faculty of Dentistry (No.4-2019) approved this study.

-Portland Cement preparation:

Barium sulphate (LobaChemie PVT.LTD, Mumbai, India) was incorporated in the ratio of $25 \%$ by weight into PC (ASEC Helwan cement, Egypt) (10).

\section{Manufacturing the Nano cements:}

Pro-Root MTA (Dentsply/Tulsa Dental, Tulsa, OK, USA) and PC were transferred into nanostructure materials using Top-down technique through a combination of various processes including fine grinding, sonication and homogenization and ultrafiltration to prevent agglomeration. Ball milling was used to produce nanomaterials by mechanical attrition in which kinetic energy from a grinding medium was transferred to material undergoing reduction. Nanomaterials were put back together 
with compaction and consolidation in an industrial scale process to form materials with enhanced properties ${ }^{(11)}$. The particle size distribution was then tested using high resolution Transmission Electron Microscope (fig 1\&2). The particles size was found to be in the range of 40-100 nms. Higher magnification showed the particles to be highly crystalline with finer crystals size. (Nano streams, Central Axis 6 October city, Cairo, Egypt).

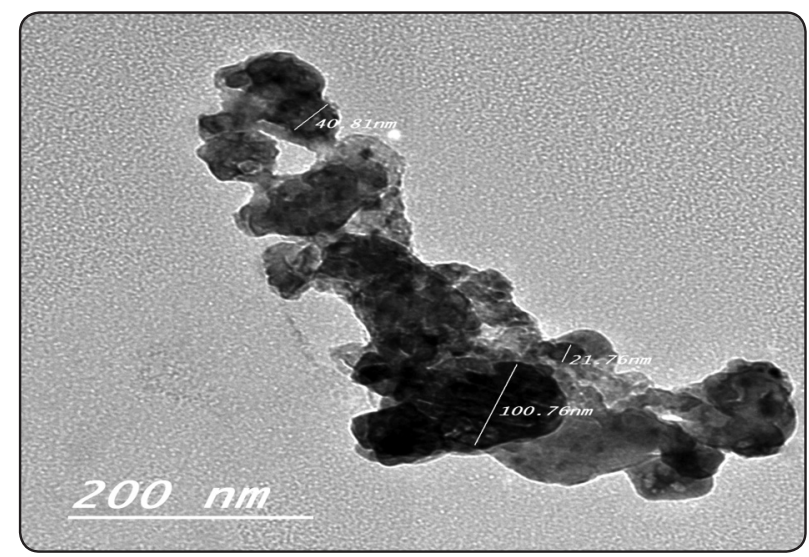

Fig. (1) TEM of MTA showing particle size less than 100nms

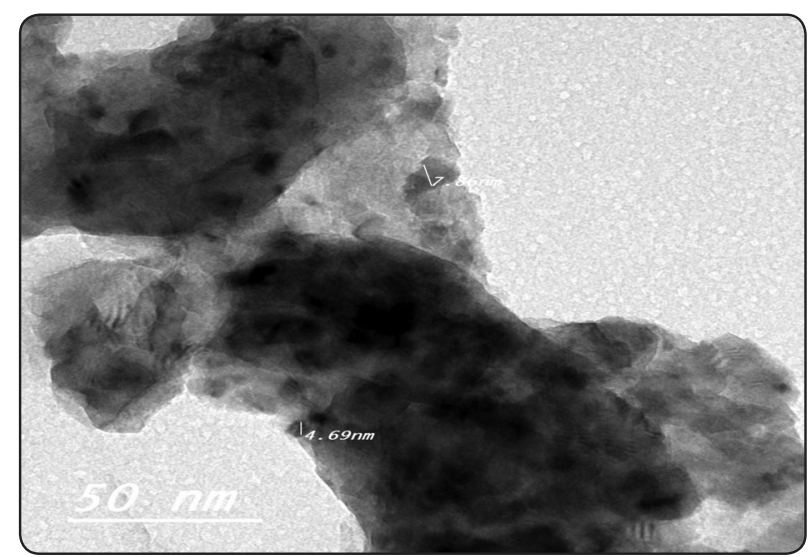

Fig. (2) TEM of Nano Portland cement showing highly crystalline particles with high magnification.

\section{Preparation of the samples:}

Forty freshly extracted single-rooted human teeth with and mature apices extracted because of orthodontic or periodontal reasons were collected. Decoronating the teeth was performed followed by sectioning of the middle third of each root perpendicular to its long axis using Isomet low- speed saw (IsoMet; 4000 microsaw Buehler Ltd., Lake Bluff, NY, USA) with continuous water irrigation to get a $2.0 \pm 0.05 \mathrm{~mm}$ thickness section. The thickness of each sample was measured by Digital caliper. To ensure standardization, the canal space of each section was instrumented with a full pass of post drills (Exacto, Angelus, Londrina, PR Brazil) to get $1.3 \mathrm{~mm}$ diameter cavities.

The samples were placed in $\operatorname{EDTA}(17 \%)$ for 3 minutes then they were immersed in $\mathrm{NaOCl}(1 \%)$ for the same time. Finally, the samples were washed with distilled water and dried immediately ${ }^{(8)}$.

The root sections were divided into four groups ( $n=10)$, the cavities were then filled as follow:

Group (I): ProRoot MTA (Dentsply/Tulsa Dental, Tulsa, OK, USA) (PMTA)

Group (II): Portland cement (PC)

\section{Group (III): Nano Pro Root MTA (NPMTA)}

\section{Group (IV): Nano PC (NPC)}

The powder/water ratio of the experimental cements:

The MTA was mixed according to the manufacturer's instructions. The prototype cements were mixed at distilled water to cement ratio of $0.30^{(10)}$

Closed plastic container was used to preserve each group separately. Gauze pieces soaked in saline were used to wrap all specimen that were kept for a period of 3 days at $37^{\circ} \mathrm{C}$ temperature to set. To keep a sufficiently moist environment inside the closed plastic container, pieces which were saline-soaked were replaced every day.

\section{PUSH-OUT TEST}

The Universal testing machine (Instron testing machine, Model3345, ITW, MA, USA) was used for the push-out bond strength evaluation of the samples. A custom-made plate was used to place the 
samples, they were aligned to the hole in the centre of the plate. This allowed the cylindrical stainlesssteel plunger (1-mm in diameter) to pass through freely under a constant compressive force at a speed of $1 \mathrm{~mm} / \mathrm{min}$. The plunger had a flat tip which was positioned to contact the test material only. The maximum load utilised on the tested materials was recorded in Newton $(\mathrm{N})$ at the time of dislodgment by a computer. The bond strength was calculated in mega pascal (MPa), the value detected was divided by the adhesion surface area of the root canal filling as follow:

Push-out bond strength $(\mathrm{MPa})=$ Maximum load $(\mathrm{N}) /$ Adhesion area $\left(\mathrm{mm}^{2}\right)$

Adhesion area $=2 \Pi r h$

$\Pi=3.14$.

$\mathrm{r}=$ Root canal radius in millimetres

$\mathrm{h}=$ Root dentin specimen thickness in millimetres.

All the samples were examined under Stereomicroscope (SZTP; Olympus Optical Co., Tokyo, Japan) at magnification $\times 25$ to detect the bond failure nature. Each sample was placed in one of three categories of failure modes: cohesive failure which was the failure that took place within the cement, adhesive failure which was the failure that took place at the cement/ dentin interface and mixed failure.

\section{Statistical analysis}

Shapiro-Wilk test and Kolmogrov-Smirnov test were used to evaluate the data for normality. Kruskal Wallis test was used for groups' comparison and Mann-Whitney $\mathrm{U}$ test was used for pairwise comparison. Chi square test was used to detect if there is a significant association between the material type and the mode of bond failure. The level of significance was set at 0.05. Statistical analysis was performed using IBM ${ }^{\circledR}$ SPSS ${ }^{\circledR} 20$ (SPSS, Inc., IBM Corporation, NY, USA).

\section{RESULTS}

\section{Push-out bond strength:}

The push-out bond strength mean values and the standard deviation of each material are presented in (Table 1). The mean push-out bond strength \pm standard deviation in MPa values of PMTA, PC, NPMTA and NPC were $1.33 \pm 0.4,0.91 \pm 0.34,1.1$ \pm 0.39 and $2.22 \pm 0.43$, respectively. A statistically significant difference was detected between the four experimental groups $(\mathrm{p}=0.007)$.

Pair wise comparison between the tested materials using the Mann-Whitney tests showed that there was no significant difference between the means of PMTA and that of PC and NPMTA groups ( $\mathrm{p}=0.529)$, but the mean of NPC was significantly higher than the rest of the group $(\mathrm{p}=0.012)$. (Table 2$)$

\section{Mode of failure:}

Chi square test revealed no significant association was revealed by between the tested materials and type of failure ( $\mathrm{p}=0.254)$. PMTA, PC and NPMTA groups showed a majority of adhesive failure, while NPC showed a majority of mixed type failure. Table (3)

TABLE (1): Mean, standard deviation and results of Kruskal-Wallis test for comparison of push-out bond strength of the four groups

\begin{tabular}{|c|c|c|c|c|c|}
\hline & PMTA Group & PC Group: & NPMTA Group & NPC Group & P value \\
\hline Mean \pm SD & $1.33 \pm 0.40$ & $0.91 \pm 0.34$ & $1.10 \pm 0.39$ & $2.22 \pm 0.43$ & $0.007 *$ \\
\hline
\end{tabular}

*significant at $p \leq 0.05$ 
TABLE (2): Mann-Whitney tests results for pair wise comparison of the values of push out bond strength:

\begin{tabular}{|l|l|}
\hline Comparative Groups & P - Value \\
\hline PMTA Group- PC Group & 0.144 \\
\hline PMTA Group- NPMTA Group & 0.529 \\
\hline PMTA Group- NPC Group & $0.012^{*}$ \\
\hline PC Group - NPC Group & $0.012^{*}$ \\
\hline PC Group - NPMTA Group & 0.529 \\
\hline NPMTA Group -NPC group & $0.012^{*}$ \\
\hline
\end{tabular}

\section{Mode of failure:}

Chi square test revealed no significant association was revealed by between the tested materials and type of failure $(p=0.254)$. PMTA, PC and NPMTA groups showed a majority of adhesive failure, while NPC showed a majority of mixed type failure. Table (3)

TABLE (3): Frequencies (N), percentages (\%) and the result of Chi square test for comparison of the mode of failure between the four groups:

\begin{tabular}{|l|c|c|c|c|c|c|c|c|c|}
\hline & \multicolumn{2}{|c|}{ PMTA Group } & \multicolumn{2}{c|}{ PC Group } & \multicolumn{2}{c|}{ NPMTA Group } & \multicolumn{2}{c|}{ NPC Group } & \multirow{2}{*}{ P- Value } \\
\cline { 2 - 9 } & $\mathrm{N}$ & $\%$ & $\mathrm{~N}$ & $\%$ & $\mathrm{~N}$ & $\%$ & $\mathrm{~N}$ & $\%$ & \\
\hline Adhesive & 5 & $50 \%$ & 6 & $60 \%$ & 6 & $60 \%$ & 3 & $30 \%$ & \\
\hline Cohesive & 3 & $30 \%$ & 3 & $30 \%$ & 2 & $20 \%$ & 1 & $10 \%$ & 0.254 \\
\hline Mixed & 2 & $20 \%$ & 1 & $10 \%$ & 2 & $20 \%$ & 6 & $60 \%$ & \\
\hline
\end{tabular}

\section{DISCUSSION}

Nano dentistry is a developing field that shows a strong potential in opening a new research work avenue in dentistry. The use of nanoparticles appears promising for their ability to improve the properties of the material. The unique characteristics of the nanoparticles are attributed to their size, with a diameter of $100 \mathrm{~nm}$ or less, providing a larger contact surface area and charge density than the bulky powder ${ }^{(12)}$. This study intended to develop new nano-based cements from PMTA and PC and to investigate their push-out bond strength in comparison with (their parent) PMTA and PC.

The bond strength of retrograde filling materials is considered a significant factor in the clinical practice. These materials are required to stay in place under dislodging forces, as mechanical stress resulting from tooth function ${ }^{(13)}$. Different procedures have been used to evaluate the adhesive properties and the bond strength of restorative materials to the dentin including tensile, shear and push-out bond strength tests. The push-out bond strength test, which was applied in our study, gives practical, efficient and reliable results ${ }^{(13 \& 14)}$

For standardization, the cements were tested after similar period of time started since mixing. The 3-days interval was considered suitable in minimizing the effect of the difference in setting time of the tested materials. The materials were incubated in moist environment, since calciumsilicate based materials reveal higher strength values when placed in a moist environment for 2-7 days, rather than only $4 \mathrm{~h}$. Gancedo-Caravia \& GarciaBarbero in $2006{ }^{(15)}$ showed that ProRoot MTA push-out strength was increased by the humidity.

The present study showed similarity in the bond strength results of PMTA to PC and NPMTA. These findings came in accordance with Iacono et al (16) and Amoroso-Silva et al. ${ }^{(17)}$ who attributed the push-out bond strength similarity of MTA and PC to their similar chemical configuration. 
The high sealing ability of calcium silicate-based materials is mainly attributed to their bioactivity and apatite-formation capacity (18) and limited amount of expansion upon setting. The mean value for expansion at 24 hours was noted to be $1.02 \%$ for Grey MTA, $0.29 \%$ for PC, in water immersion ${ }^{(19)}$. Camilleri et al ${ }^{(20)}$ showed that hydration of both MTA and PC initially forms calcium hydroxide and calcium silicate hydrate gel that finally change into a poorly porous solid and crystallized gel. The calcium silicate ratio is reduced because of the calcium precipitate formation. Then, the precipitated calcium produces Calcium hydroxide, which is the reason of the high alkalinity level of the cement after hydration. Since the dicalcium silicate hydration rate is lower in rate than the tricalcium silicate rate, storing MTA and PC in a wet environment improves their retention characteristics, flexural strength and push-out strength with the passage of time. This could be explained by the elongated maturation process due to the formation of passivating trisulfate layer over hydrating crystals of MTA ${ }^{(21)}$.

Additionally, the hydration of calcium silicate based materials results in hybrid layer formation between dentin and MTA. ${ }^{(22,23)}$ It has been documented that the hybrid layer formation along with intratubular mineralization may influence the MTA push-out bond strength ${ }^{(24)}$. Also, the formed Hydroxy-apatite (HA) crystals fill the microscopic gaps between dentin and the restorative material causing chemical bonding ${ }^{(23)}$.

Also, PMTA and NPMTA may show better hydration process, due to possessing tricalcium aluminate (C3 A).The authors mentioned that tricalcium aluminate can help the formation of required silicate phase. "flash set" or "instantaneous set" procedure occurs by the reaction of this element (C3 A) with water and the creation of $\mathrm{Ca}_{2} \mathrm{AlO}_{3}(\mathrm{OH})$ $\mathrm{nH}_{2} \mathrm{O}$, which is capable of enhancing the strength of the mixed cement ${ }^{(25)}$. While other investigations $(26,27)$ stated that tricalcium aluminate has a major influence on the strength of calcium silicate-based cements and if it is absent the cement strength may be affected and its setting time could be delayed.

On the other hand, NPC showed the highest values of bond strength when compared to the other three groups. It is believed that the nano formulation was able to alter the size of the constituents of PC which lead to the powder surface area increase. The micro hardness of the material might have increased due to the nano-modification in addition to the uniform distribution of particles ${ }^{(9)}$.

The bond failure of the tested materials was investigated in this study. PMTA, PC and NPMTA presented a majority of adhesive failure at the restorative/dentin interface. This came in conjugation with Vanderweele et al. ${ }^{(28)}$, Saghiri et al. (29), and Shahi et al. (13) that showed that adhesive type of failure was found in MTA-dentin bond. The cement penetration into dentinal tubules could have been prevented due to the particle size, producing adhesive failures ${ }^{(30)}$. While, NPC showed predominantly mixed type of failure which could be attributed to incomplete setting of the material that led to limited physical properties. Also, the difference in the size of the particles of the tested cements played a major role in their ability of penetration and the consequent interlocking of NPC within the dentinal tubules ${ }^{(8)}$. Since, bond strength is formed from the union between two surfaces having different molecular compositions as a result of mechanical, chemical, or physical forces ${ }^{(31)}$, thus, different modes of failure were caused by difference in the material structural performance.

Though, the invitro approach of push-out cannot duplicate the invivo environment, the results of the study can provide information that helps in the development of a novel root-end filling/perforation repair material with suitable physical and adhesive properties. NPMTA showed comparative retention when compared to PMTA. NPC showed higher resistance to dislodgment forces when compared to PMTA and NPMTA. Both nano-formulated materials need further researches regarding other physical properties. 


\section{CONCLUSION}

The force needed for NPMTA displacement is similar to PMTA and PC and significantly lower than the force required to displace NPC.

Adhesive failure was the type of bond failure shown in NPMTA, PMTA and PC, while NPC showed mixed failure. Therefore, for better understanding of adhesion characteristics of the newly introduced nano-formulations of the cements, further investigations are recommended.

\section{Conflict of interest}

The authors declare that there is no conflict of interest related to this research and there is no financial involvement with any commercial organization in the subject or materials discussed in this manuscript.

\section{Highlights key points:}

- The bond strength of the retrograde filling materials plays a significant role in their adaptation to the dentinal wall, ability to seal the root canal and resistance to dislodging forces.

- The usage of nanoparticles appears promising for their ability to enhance the properties of the material.

- Current study showed that the NPC exhibited the highest value of push-out strength followed by PMTA and the NPMTA cements, while PC showed the least value of push-out bond strength.

\section{REFERENCES}

1. Torabinejad M, Watson TF, Pitt Ford TR. Sealing ability of a mineral trioxide aggregate when used as a root end filling material Journal of Endodontics. 1993; 19:591-595.

2. Torabinejad M, Hong CU, Lee SJ, Monsef M, Pitt Ford TR. Investigation of mineral trioxide aggregate for rootend filling in dogs. Journal of Endodontics. 1995; 21, 603-8.

3. Oliveira MG, Xavier CB, Demarco FF, Pinheiro AL, Costa AT, Pozza DH. Comparative chemical study of MTA and Portland cements. Braz Dent J. 2007; 18(1):3-7.
4. Sanchez F, Sobolev K. Nanotechnology in concrete-a review. Construction and Building Materials 2010; 24:2060-2071.

5. Commission E. Commission Recommendation of 18 October 2011 on the Definition of Nanomaterial (2011/696/EU). Official Journal of the European Communities: Legis 2011.

6. Tennis P, Jennings HM. A model for two types of calcium silicate hydrate in the microstructure of Portland cement pastes Cement and Concrete Research 2000: 6, 855-863.

7. Komabayashi T, Spangberg LS Particle size and shape analysis of MTA finer fractions using Portland cement Journal of Endodontics 2008: 6, 709-711.

8. Saghiri MA, Garcia-Godoy F, Gutmann JL, Lotfi M, Asatourian A, Ahmadi H. Push-out bond strength of a nanomodified mineral trioxide aggregate Dental Traumatology 2013; 29: 323-327.

9. Saghiri MA, Asgar K, Lotfi M, Garcia-Godoy F. Nanomodification of mineral trioxide aggregate for enhanced physiochemical properties. Int Endod J 2012; 45:979-88

10. Camilleri J. Evaluation of the physical properties of an endodontic Portland cement incorporating alternative radiopacifiers used as root-end filling material. International Endodontic Journal 2010: 43, 231-240.

11. V.M.Arole. Fabrication of nanomaterials by Top-Down and Bottom-Up approaches-An overview. Journal of Advances in Applied Sciences and Technologies 2014; 1: 89-93.

12. ElKateb Walid M., Massoud Ahmed G., Mokhless Nayera A, Shalaby Thanaa I. Measurement of Tubular Penetration Depth of Three Types of Nanoparticles Mixed With Endodontic Sealer Using Scanning Electron Microscope (An In Vitro Study). J Am Sci2015; 11(11):111-122.

13. Shahi S, Rahimi S, Yavari HR, Samiei M, Janani M, Bahari $\mathrm{M}$, et al. Effects of various mixing techniques on pushout bond strengths of white mineral trioxide aggregate. $\mathrm{J}$ Endod. 2012;38:501-4

14. Shokouhinejad N, Nekoofar MH, Iravani A, Kharrazifard MJ, Dummer PM. Effect of acidic environment on the pushout bond strength of mineral trioxide aggregate. Journal of Endodontics 2010; 36:871-874.

15. Gancedo-Caravia L, Garcia-Barbero E. Influence of humidity and setting time on the push-out strength of mineral trioxide aggregate obturations. Journal of Endodontics 2006; 32:894-896. 
16. Iacono F, Gandolfi MG, Huffman B, Sword J, Agee K, Siboni F, Tay F, Prati C, Pashley D. Push-out strength of modified Portland cements and resins. American Journal of Dentistry 2010; 23: 43-46.

17. Amoroso-Silva PA, Marciano MA, Guimarães BM, Duarte MA, Sanson AF, Moraes IG. Apical adaptation, sealing ability and pushout bond strength of five root-end filling materials. Brazillian Oral Research 2014; 28: 40-45.

18. Gandolfi MG, Taddei P, Siboni F, Modena E, Ginebra MP, Prati C. Fluoride-containing nanoporous calcium-silicate MTA cements for endodontics and oral surgery: Early fluorapatite formation in a phosphate-containing solution. International Endodontic Journal 2011; 44:938-949

19. Storm B, Eichmiller FC, Tordik PA, Goodell GG. Setting expansion of gray and white mineral trioxide aggregate and Portland cement. Journal of Endodontics 2008;34 (1):80-2.

20. Camilleri J, Montesin FE, Papaioannou S, McDonald F, Pitt Ford TR. Biocompatibility of two commercial forms of mineral trioxide aggregate. International Endodontic Journal 2004; 37:699-704.

21. Dammaschke T, Gerth HU,Zuchner H, Schafer E. Chemical and physical surface and bulk material characterization of white ProRoot MTA and two Portland cements. Dental Materials 2005; 21:731-738.

22. Asgary S, Eghbal MJ, Parirokh M, Ghoddusi J, Kheirieh S, Brink F. Comparison of mineral trioxide aggregate's composition with Portland cements and a new endodontic cement. Journal of Endodontics 2009; 35:243-250.

23. Sarkar NK, Caicedo R, Ritwik P, Moiseyeva R, Kawashima I. Physicochemical basis of the biologic properties of mineral trioxide aggregate. Journal of Endodontics 2005; 31:97-100.
24. Bozeman TB, Lemon RR, Eleazer PD. Elemental analysis of crystal precipitate from gray and white MTA. Journal of Endodontics 2006; 32:425-8.

25. Park JW, Hong SH, Kim JH, Lee SJ, Shin SJ. X-Ray diffraction analysis of white ProRoot MTA and Diadent BioAggregate. Oral Surg Oral Med Oral Pathol Oral Radiol Endod 2010; 109:155-158.

26. Liu WN, Chang J, Zhu YQ, Zhang M. Effect of tricalcium aluminate on the properties of tricalcium silicate-tricalcium aluminate mixtures: setting time, mechanical strength and biocompatibility. International Endodontic Journal 2011; 44:41-50.

27. Shie MY, Chang HC, Ding SJ. Effects of altering the Si/ Ca molar ratio of a calcium silicate cement on in vitro cell attachment. International Endodontic Journal 2012; 45:337-345

28. Vanderweele RA, Schwartz SA, Beeson TJ. Effect of blood contamination on retention characteristics of MTA when mixed with different liquids. Journal of Endodontics 2006; 32:421-424.

29. Saghiri MA, Shokouhinejad N, Lotfi M, Aminsobhani M, Saghiri AM. Push-out bond strength of mineral trioxide aggregate in the presence of alkaline $\mathrm{pH}$. Journal of Endodontics 2010; 36:1856-9.

30. Komabayashi T, Spangberg LSW. Comparative analysis of the particle size and shape of commercially available mineral trioxide aggregate and Portland cement: a study with a flow particle image analyzer. Journal of Endodontics 2008; 34:94-8.

31. Erickson RL. Surface interactions of dentin adhesive materials. Operative Dentistry. 1992; Suppl 5:81-94. 